\title{
Bifurcation of Jovian magnetotail current sheet
}

\author{
P. L. Israelevich and A. I. Ershkovich \\ Department of Geophysics and Planetary Sciences, The Raymond and Beverly Sackler Faculty of Exact Sciences, Tel Aviv \\ University, Ramat Aviv, Israel
}

Received: 31 March 2006 - Accepted: 17 May 2006 - Published: 3 July 2006

\begin{abstract}
Multiple crossings of the magnetotail current sheet by a single spacecraft give the possibility to distinguish between two types of electric current density distribution: single-peaked (Harris type current layer) and double-peaked (bifurcated current sheet). Magnetic field measurements in the Jovian magnetic tail by Voyager-2 reveal bifurcation of the tail current sheet. The electric current density possesses a minimum at the point of the $B_{x}$-component reversal and two maxima at the distance where the magnetic field strength reaches $50 \%$ of its value in the tail lobe.
\end{abstract}

Keywords. Magnetospheric physics (Current systems; Magnetotail; Planetary magnetospheres)

\section{Introduction}

Satellite observations have shown that geotail current sheet often exhibits two off-centered maxima of electric current density. The first evidence for a bifurcated current sheet was reported by Sergeev et al. (1993) using a dual satellite system (ISEE). Statistical studies of Geotail current sheet crossings in the distant $\left(\sim 50-125 R_{E}\right)$ tail (Hoshino et al., 1996) gave further evidence for the existense of bifurcated current sheets. Direct measurements of electric current density by the Cluster system of satellites (e.g. Runov et al., 2003, 2004, 2005; Sergeev et al., 2003) revealed a number of double peaked current sheet crossings and thereby stimulated theoretical and model studies of the current sheet structure and possible causes of its bifurcation (e.g. Sitnov et al., 2003; Zelenyi et al., 2003; Ricci et al., 2004; Genot et al., 2005; Camporeale and Lapenta, 2005). A first statistical analysis of bifurcated current sheets (Asano et al., 2005) has shown that they are a rather common feature of the Earth's magnetosphere. Therefore, it is natural to expect that this phenomenon exists in the magnetotail of any planet possessing an intrinsic magnetic field and to search for its presence. Single-satellite magnetic field measurements in the magne-

Correspondence to: P. L. Israelevich

(peteri@post.tau.ac.il) tospheres of giant planets do not allow one to calculate the electric current density. Hoshino et al. (1996) used the histogram of the occurrence frequency of the $B_{x}$-component of the magnetic field. The histogram should possess near $B_{x}=0$ a pronounced maximum for the case of a bifurcated current sheet and a minimum for the case of a single-peaked current sheet. While multi-satellite Cluster measurements have been given credit for pioneering statistical analysis of the bifurcated current sheet, the method used by Hoshino et al. (1996) for Geotail data can hardly be applied to magnetotails of the outer planets because of relatively poor (as compared to Geotail) statistics. In this paper, we propose a simple method, enabling us to reveal current sheet splitting from single-satellite data. We analyze Voyager-2 magnetic field data in the Jovian magnetic tail and show that, at least in one case, the current sheet has an off-centered double peak structure.

\section{Method}

Using multiple crossings of the magnetotail current sheet, it is possible to distinguish between the single-peaked and bifurcated layers in the following way. Let us use the coordinate system $X, Y, Z$, in which $X Y$ is the plane of the current sheet, the $Y$-axis is antiparallel to the current direction and $z$ $=0$ corresponds to the current sheet center, i.e. $B_{x}=0$ at $z=0$. Generally, the magnetic field in the tail is a certain function of position, the set of solar wind parameters $P_{i}$ (like density, velocity, etc.), and, maybe, the time:

$\mathbf{B}=\mathbf{B}\left(x(t), y(t), z(t), P_{i}(t) ; t\right)$.

However, it is obvious that in the vicinity of the current sheet (i.e. close to $z=0$ ), the dependence of $\mathbf{B}$ on $z$ is dominant (as compared with the dependencies on other parameters). The dependence of $\mathbf{B}$ on changing solar wind parameters can also be neglected if the time period of multiple current sheet crossings is short. Therefore,

$B_{x}=B_{x}\left(x(t), y(t), z(t), P_{i}(t) ; t\right) \approx B_{x}(z)$

can be considered as a measure of the satellite distance from the point of $B_{x}$-component reversal $(z=0)$. (Of course, if $B_{x}$ increases monotonously with $z$.) 


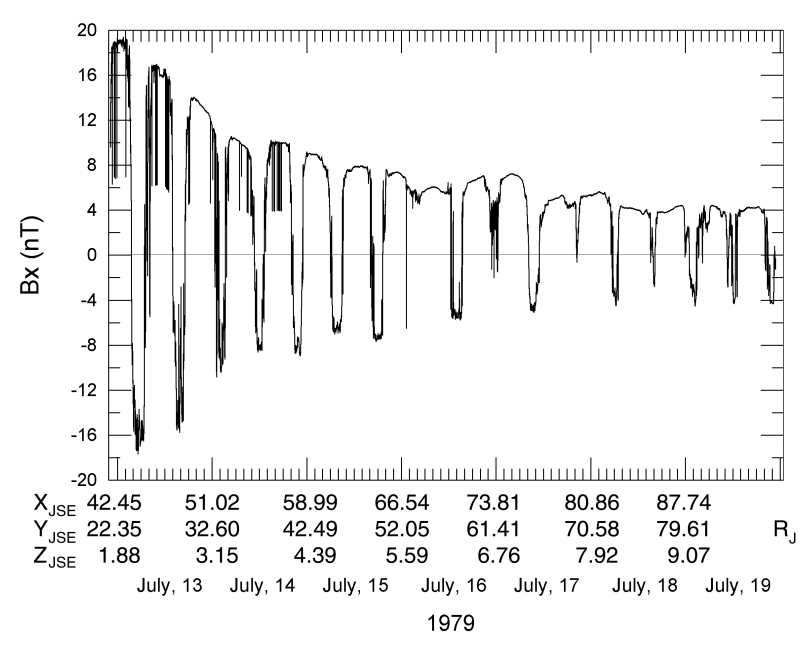

Fig. 1. Crossings of Jovian magnetotail current sheet by Voyager-2: $B_{x}$-component during 13-19 July 1979.

Multiple current sheet crossings occur because of changing satellite position, $\mathrm{z}$, with time, due to the tail flapping motion. Thus, the observed time series of magnetic field measurements is $B_{x}(t) \approx B_{x}(z(t))$.

Hence, the measurable full derivative $d B_{x} / d t$ is

$\frac{d B_{x}}{d t}=\frac{\partial B_{x}}{\partial z} \frac{d z}{d t}$.

The electric current density is proportional to $\partial B_{x} / \partial z$, and $d z / d t$ is the vertical component of the velocity of the tail motion. The measurements provide us with sets of simultaneous values of $d B_{x} / d t$ and $B_{x}$. Averaging $d B_{x} / d t$ for given values of $B_{x}$ (which is equivalent to the averaging over time) we can find the functional dependence of $<d B_{x} / d t>$ on $B_{x}$, i.e. on the distance from the current sheet center. Obviously, $<d z / d t>\approx 0$ for multiple crossings of the current sheet, since they occur because of quasi-periodical oscillations of the tail in the $z$-direction. Hence, $\left\langle d B_{x} / d t>\approx 0\right.$. On the other hand, $<|d z / d t|>\neq 0$ and has a maximum near $z=0\left(B_{x}=0\right)$. (The maximum may be somewhat shifted with respect to $z=0$ because of the global motion of the current sheet in the $z$ direction, but the very existence of a multiple crossing means that the flapping motion is much faster than the average motion of the sheet, so the shift is small.) The derivative $\partial B_{x} / \partial z$ is always positive because of the choice of coordinate system, so that

$\left\langle\left|\frac{d B_{x}}{d t}\right|\right\rangle=\frac{\partial B_{x}}{\partial z}\left\langle\left|\frac{d z}{d t}\right|\right\rangle$

and the dependence of $\left\langle\left|d B_{x} / d t\right|>\right.$ on $B_{x}$ enables us to distinguish between different types of distribution of the electric current density. Indeed, for the single-peaked current sheet $\partial B_{x} / \partial z$ has a maximum at $z=0\left(B_{x}=0\right)$ and $<\left|d B_{x} / d t\right|>$ will also have a maximum with $B_{x}=0$. On the contrary, if

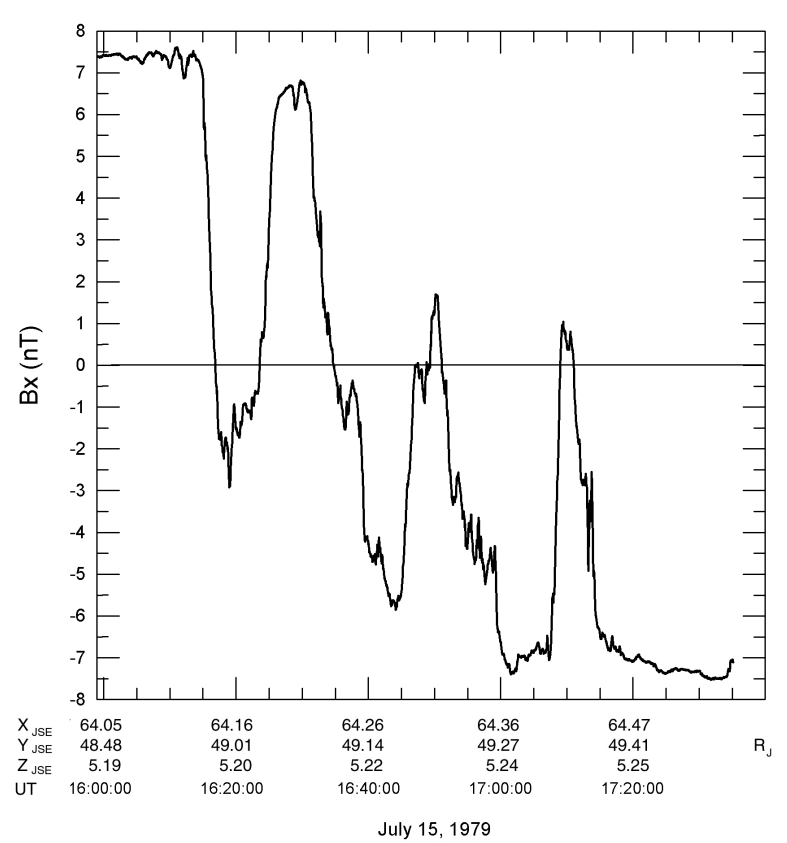

Fig. 2. $B_{x}$-component during multiple crossing of the Jovian magnetotail current sheet on 15 July 1979, at 16:00-17:40.

$<\left|d B_{x} / d t\right|>$ possesses two off-centered maxima and the minimum at $B_{x}=0$, this means that $\partial B_{x} / \partial z$ also has two maxima and the current sheet is bifurcated. Of course, these are two extreme cases, and it is impossible to judge the current sheet structure, if the maxima of $\left\langle\left|d B_{x} / d t\right|>\right.$ are not clearly defined.

\section{Multiple crossing of the Jovian magnetotail current sheet}

During the Voyager 2 encounter with Jupiter in July 1979, the magnetotail current sheet was crossed many times (Behannon et al., 1981). Figure 1 shows the $B_{x}$-component of the magnetic field for the period 13-19 July 1979. The spacecraft appeared periodically in the northern and southern lobes of the Jovian magnetic tail because of global tail motion, due to the rotation of the tilted magnetic dipole of the planet. One can see, however, that the strength of the lobe field changed mainly with the distance from the planet, and, therefore, it was indeed possible to neglect the dependence of the lobe field on varying solar wind parameters. The individual passes from the northern to the southern tail lobe and vice versa sometimes exhibit multiple crossings of the magnetotail current sheet, due to quasi-periodical oscillations of the current sheet position on the background of slow periodic variations because of the rotation of the tilted magnetic dipole. Figure 2 shows such a multiple crossing which occured on 15 July 1979, at 16:00-17:40 at the distance of $65 R_{J}$ in the tail. During this period, $B_{x}$ changed 


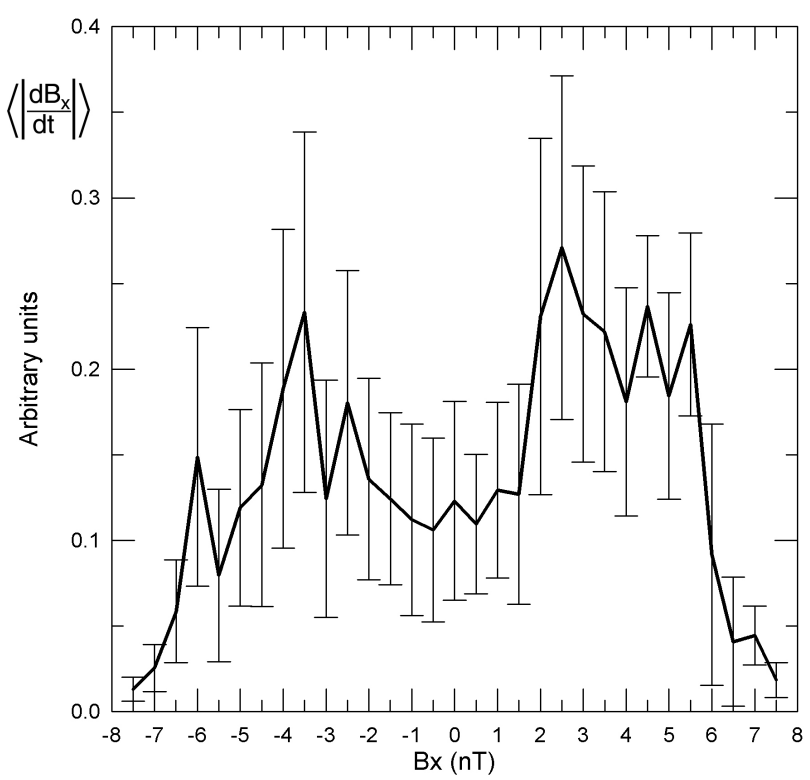

Fig. 3. Averaged $<\left|d B_{x} / d t\right|>$ depending on $B_{x}$.

its sign 7 times. The full derivative, $d B_{x} / d t$, was calculated $\left[B_{x}(t+\Delta t)-B_{x}(t-\Delta t)\right] / 2 \Delta t$ and was put into correspondance to $B_{x}(t)$. ( $\Delta t$ was equal to $1.92 \mathrm{~s}$.) The obtained values of $\left|d B_{x} / d t\right|$ were averaged in $0.5 \mathrm{nT}$ bins of the corresponding $B_{x}$ values. The resulting $<\left|d B_{x} / d t\right|>$ is shown in Fig. 3 as a function of $B_{x}$. Vertical bars show r.m.s. values for each bin. Distribution of $<\left|d B_{x} / d t\right|>$ exhibits two distinct maxima and the minimum at $B_{x}=0$, i.e. at $z=0$. Thus, we arrive at the conclusion that on 15 July 1979, at 16:0017:40, Voyager 2 crossed the bifurcated current sheet in the Jovian magnetic tail. For other current sheet crossings shown in Fig. 1, the maxima of $\left\langle\left|d B_{x} / d t\right|>\right.$ are not distinct enough in order to judge the sheet structure.

\section{Conclusions}

One undoubted case of a split current sheet crossing is found in Voyager-2 data. The structure of the bifurcated current sheet in the Jovian magnetic tail is similar to that of the bifurcated current sheets observed in the geomagnetic tail by Cluster satellites. The electric current density has maxima at the distance from the $B_{x}$-component reversal, where the magnetic field strength reaches $50 \%$ of the magnetic field in the tail lobe.
Acknowledgements. Voyager-2 magnetic field data were obtained from Planetary Data System http://pds.jpl.nasa.gov.

Topical Editor I. A. Daglis thanks L. Zelenyi for his help in evaluating this paper.

\section{References}

Asano, Y., Nakamura, R., Baumjohann, W., Runov, A., Vörös, Z., Volwerk, M., Zhang, T. L., Balogh, A., Klecker, B., and Rème, H.: How typical are atypical current sheets?, Geophys. Res. Lett, 32, L03108, doi:10.1029/2004GL021834, 2005.

Behannon, K. W., Burlaga, L. F., and Ness, N. F.: The Jovian magnetotail and its current sheet, J. Geophys. Res., 86 (A10), 83858401, 1981..

Camporeale, E. and Lapenta, G.: Model of bifurcated current sheets in the Earth's magnetotail: equilibrium and stability, J. Geophys. Res., 110, A07206, doi:10.1029/2004JA010779, 2005.

Genot, V., Mottez, F., Fruit, G., Louarn, P., Sauvaud, J. A., and Balogh, A.: Bifurcated current sheet: model and Cluster observations, Planet. Space Sci., 53, 229-235, 2005.

Hoshino, M., Nishida, A., Mukai, T., Saito, Y., Yamamoto, T., and Kokubun, S.: Structure of plasma sheet in magnetotail: Doublepeaked electric current sheet, Geophys. Res., 101, 2477524 786, 1996.

Ricci, P., Lapenta, G., and Brackbill, J. U.: Structure of the magnetotail current: Kinetic simulation and comparison with satellite observations, Geophys. Res. Lett., 31, L06801, doi:10.1029/2003GL019207, 2004.

Runov, A., Nakamura, R., Baumjohann, W., Zhang, T. L., Volwerk, M., Eichelberger, H.-U., and Balogh, A.: Cluster observation of a bifurcated current sheet, Geophys. Res. Lett., 30, 1036, doi:10.1029/2002GL016136, 2003.

Runov A., Sergeev, V., Nakamura, R., Baumjohann, W., Vörös, Z., Volwerk, M., Asano, Y., Klecker, B., Rème, H., and Balogh, A.: Propeties of a bifurcated current sheet observed on 29 August 2001, Ann. Geophys., 22, 2535-2540, 2004.

Runov, A., Sergeev, V., Nakamura, R., Baumjohann, W., Zhang, T. L., Asano, Y., Volwerk, M., Vörös, Z., Balogh, A., and Rème, H.: Reconstruction of the magnetotail current sheet structure using multi-point Cluster measurements, Planet. Space Sci., 53, 237243, 2004.

Sergeev, V. A., Mitchell, D. G., Russell, C. T., and Williams, D. J.: Structure of the tail plasma/current sheet at $\sim 11 \mathrm{R}_{E}$ and its changes in the course of a substorm, J. Geophys. Res., 98, 17345-17365, 1993.

Sergeev, V., Runov, A, Baumjohann, W., et al.: Current sheet flapping motion and structure observed by Cluster, Geophys. Res. Lett., 30, 1327, doi:10.1029/2002GL016500, 2003.

Sitnov, M. I., Guzdar, P. N., and Swisdak, M.: A model of the bifurcated current sheet, Geophys. Res. Lett.,30, 1712, doi:10.1029/2003GL017218, 2003.

Zelenyi, L. M., Malova, H. V., and Popov, V. Yu.: Splitting of thin current sheets in the Earth's magnetosphere, JETP Lett., 78, 296299, 2003. 